\title{
EL CANCIONERO SEFARDÍ DE BULGARIA KANTIKAS VIEJAS: AVANCE DE UN CATÁLOGO Y EDICIÓN CRÍTICA
}

\author{
Susana WEICH-SHAHAK
}

Me complace proponer a la atención de colegas musicólogos y amantes de la cultura sefardí una colección anotada en la comunidad de Samokoff en Bulgaria'. Este cancionero ha llegado a mis manos a mediados de 1993, gentilmente of recido por Naamá y Eli Arié, descendientes de los recopiladores de la colección ${ }^{2}$.

\section{LOS RECOPILADORES DEL CANCIONERO DE SAMOKOFF}

La compilación del cancionero que nos ocupa fue comenzada por Rahamim Arié, nacido en 1865 y fallecido en 1916, y continuada por su hijo, Yosef Arié, nacido en 1896 y fallecido en 1963. Ambos eran miembros de la renombrada familia Arié en Samokoff, importante en la vida comunitaria de la ciudad y en su economía; prueba de su posición social es el hecho de que la casa (o palacete) que perteneciera a la familia Arié está siendo restaurada como monumento nacional.

1. Realizo mis trabajos en el marco del Centro de Investigaciones de la Música Judía de la Universidad Hebrea de Jerusalen, y he podido completar esta presentación de material gracias a la ayuda otorgada por la Subdirección General de Promoción de Investigación del Ministerio de Educación y Ciencia de España para mi proyecto en el Departamento de Antropología (Instituto de Filología) del CSIC de Madrid. Vaya aquí mi agradecimiento a estas instancias y a mis colegas por las condiciones prestadas para la realización de este estudio.

2. Al poco tiempo de entregarme este Cancionero, el matrimonio Arié me permitió fotocopiar otro importante documento: se trata de un diario historiado de la familia Arić, escrito en judeoespañol, en letras latinas; este diario es una fuente de rarovalor como re flejo de la sociedad sefardí de los Balcanes, ya que ofrece riquísimas informaciones sobre aspectoscostumbristas, rituales, sociales y económicos de su tiempo ( último cuarto del siglo XIX hasta mediados del XX). De este documento he depositado una copia en el Instituto de Investigaciones de las Diásporas y otra en la Biblioteca Nacional y Universitaria de la Universidad Hebrea de Jerusalén. El que aquí nos ocupa, KANTIKAS VIEJAS, está catalogado en la JNUL en la Weich-Shahak "Judeo Spanish Mss. Collection", Music Department, Musical Collection, n. .: JMB 2366. 


\section{TÍTULO Y NOMBRE DEL CANCIONERO}

La colección está titulada por sus propios compiladores KANTIKAS VIEJAS, y como tal figura en letras latinas en una primera página añadida a sus hojas. A este título ha agregado Naamá Arié, en letras hebreas manuscritas cursivas, el subtítulo (que aquí traduzco del hebreo): "Canciones antiguas en ladino que fueran costumbre en la comunidad judía de Bulgaria" y, más abajo: “compilado por Rahamim Arie (1865-1916) y su hijo Yosef Arié (1896-1963)”.

\section{FORMATO Y CONTENIDO DEL CANCIONERO}

El Cancionero KANTIKAS VIEJAS consta de 115 folios mecanografiados por una sola cara; al parecer, la colección que ha llegado a nosotros es una copia en papel carbón del original mecanográfico. Hay en esta colección 109 textos, cada uno en una página, y sólo en 6 casos extendidos a la página siguiente. Pocos temas se repiten. En muchos casos se anota también la fuente del cantar: de quién se ha a oído o quién lo acostumbraba a cantar, en que ocasión se oyó, o de dónde se ha anotado, inclusive señalando si proviene de alguna otra ciudad de los Balcanes y no de la tradición local de Samokoff.

La colección que tenemos ante nosotros representa abundantemente los tres géneros poético-musicales principales del repertorio judeo-español: el Romancero, el Cancionero y las Coplas, incluyendo algunos temas desconocidos hasta ahora, así como versiones especialmente originales e interesantes de temas ya conocidos. De algunos temas conozco solamente versiones paralelas en la tradición oral, recogidas en mis encuestas con informantes sefardíes ${ }^{3}$.

\section{CRITERIOS DEL AVANCE CATALOGRÁFICO}

Se da a continuación un avance de catálogo del corpus recogido en la colección KANTIKAS VIEJAS, que ofrece:

-el número de página,

-el título que encabeza la canción en el original mecanografiado (tal como está, sin normalización ni corrección de obvios errores ortográficos, mecanográficos o de inconsistencias gramaticales, y en el mismo tipo de letras, mayúsculas o minúsculas, en que se anotaron);

-el íncipit (entre comillas) o primer verso tal como figura en el original, también sin corregir obvios errores;

-el número de estrofas (entre paréntesis), tal como están distribuidas y, generalmente, numeradas en el original, aunque esta distribución y numeración sean frecuentemente arbitra-

3. Me refiero a versiones en mi colección, catalogada en la Fonoteca Nacional (National Sound Archives) en la JNUL. Los ejemplos que se presentan llevan la signatura de su catálogo. 
rias. Doy el número entre corchetes cuando no hay numeración en el original. Indico cuántas estrofas aparecen completas y cuántas abreviadas (las que aparecen truncadas y con puntos suspensivos o con la indicación de "etc."), y si hay versos que se repiten como estribillo;

-el título convencional (en cursiva): si el tema pertenece al Romancero, según Armistead"; si a las Coplas, según E. Romero'; y si al Cancionero, usando parcialmente el trabajo de catálogo y filiación que tiene en curso J. M. Pedrosa", complementado por algunos nombres que yo he dado a ciertas canciones que estaban sin filiar, en especial aquéllas de las cuales conozco versiones orales recogidas en mis encuestas, lo que me ha facilitado la elección de título. Ha parecido preferible dejar sin titular algunos temas raros o desconocidos hasta ahora, en cuyo caso me he limitado a señalar SF, para indicar SIN FILIACION.

-el género: Romance, Copla, Canción (lírica, seriada o narrativa), o Himno y, eventualmente, y cuando se tienen datos al respecto, la función que cumple, en especial dentro del ciclo de la vida o en el festivo (de boda, de parida, etc.).

Siendo ésta una mera noticia de la existencia y presentación preliminar del documento, no se han agregado las concordancias bibliográficas con fuentes publicadas, tarea que deberá efectuarse al tiempo de preparar una edición crítica completa. También el análisis comparativo con fuentes orales documentales y con publicaciones que incluyen transcripciones musicales se dejan para dicha edición futura. Por ahora, me limito a indicar con un asterisco las canciones cuyos textos me son conocidos en versiones orales recogidas y catalogadas en la Fonoteca Nacional (NSA - National Sound Archives, Jewish National and University Library) de la Universidad Hebrea de Jerusalén. Con todo, como avance del corpus, y dentro de las limitaciones de espacio de esta publicación, se editan, como colofón, y con el objeto de poder comparar ambos estadios de la tradición, algunos textos de la colección de KANTIKAS VIEJAS, acompañados de las transcripciones (texto y música) de versiones documentales paralelas recogidas en mis encuestas de la tradición oral sefardí.

\section{AVANCE CATALOGRÁFICO}

[pag.] 1 KANTIKA VIEJA DE BODAS / entrada/ "vengach en boena ora signora de el vuestro kamino" ( 1 estrofa completa +1 abreviada, con estribillo) La novia galana Canción lírica de boda

4. Samuel G. Armistead, El Romancero judeo-español en el Archivo Menéndez. Pidal: Catálogo-indice de romances y canciones (Madrid, Gredos - Seminario Menendez Pidal: 1978).

5. Elena Romero, en colaboración con lacob M. Hassán y Leonor Carracedo, Bibliografía analítica de ediciones de coplas sefardies (BAECS) (Madrid, CSIC: 1992). Agradezco a Elena Romero su contribución a disipar algunas de mis dudas sobre las Coplas judeo-españolas.

6. He podido consultar tales trabajos, en proceso de realización, así como sus artículos ya publicados, que suponen un apreciable avance en el conocimiento y la filiación del Cancionero sefardí, gracias a la amable disposición de mi amigo, José Manuel Pedrosa, a quien además agradezco la lectura e indicaciones aportadas a este trabajo. 
[pag.] 2 KANTIKA VIEJA DE BODAS / kidouchim/ "signora novia abachech abacho non poedo non poedo $*$ ( 1 estrofa completa +3 abreviadas, con estribillo) La espera del novio Canción seriada de boda

[pag.] 3 KANTIKA VIEJA DE BODAS / despoes de kidouchim/ "oh, ke reloumbror de novia galana" * ( 2 estrofas completas +1 abreviada, con estribillo) La novia galana Canción seriada de boda

[pag.] 4 KANTIKA VIEJA DI BODAS / despoes de kidouchim/ "salgach madre afoera al kortijo" * ( 1 estrofa completa +3 abreviadas, con estribillo) La salida de la madre Canción seriada de boda

[pag.] 5 KANTIKA VIEJA DE BODA / kidouchim/ "en la entrada de la poerta" * (2 estrofas completas +3 abreviadas, con estribillo) $S F$ (Sin filiación) Canción lírica de boda

[pag.] 6 KANTIKA VIEJA DE BODAS / achugar sevan/ "anio de oro mandelé azer, para la ija de onra" ( 1 estrofa completa +3 abreviadas, con estribillo) Los regalos de oro Canción seriada de boda

[pag.] 7 KANTIKA DE BODA DE LAS PLEVNALIAS / dia de sevan i de achugar/ "a si biva la koshoegra" * (10 estrofas completas: 5 son repeticiones del estribillo) El regateo de las consuegras Canción seriada de boda

[pag.] 8 KANTIKA VIEYA DE BODAS /houpa/ "intché el tas de alhabaka" * (2 estrofas completas, con estribillo) El baile de la boda Canción seriada

[pag.] 9 KANTIKA VIEYA DE BODAS /houpa/ "Avri mi planeta ke ya amanissio" * (4 estrofas) Todos son inconvenientes Canción seriada de boda

[pag.] 10 VIEYA KANTIKA DE BODAS /houpa/ "kouchiniko de alhavaka la kolor aresendera" (5 estrofas) $S F+$ La novia demudada Canción lírica de boda

[pag.] 11 KANTIKA VIEJA DE BODAS /houpa/ "a levanteme ouna manianika" * (1 estrofa completa +1 abreviada, con estribillo) La madrugada de la novia Canción lírica de boda

[pag.] 12 KANTIKA VIEYA DE BODAS /komida/ "estas mezas son del vino" * (1 estrofa completa +1 abreviada, con estribillo) Las casas nobles Canción lírica de boda

[pag.] 13 KANTIKA DE BODA /houpa/ "demandalde a la voestra novia" * (1 estrofa completa y 1 abreviada, con estribillo) Las prendas de la novia Canción acumulativa de boda

[pag.] 14 KANTIKA VIEJA DE BODAS /espartission/ "kon muntcha lessensia, madre boena" * (6 estrofas) La nueva familia de la novia + La despedida de la novia $+S F+$ El perdón a la madre Canción lírica de boda

[pag.] 15 KANTIKA DE BODAS /konsejos ala novia/ "ke dizi la pera, ke eskudyé a su esfoegra" * ( 2 estrofas completas +3 abreviadas) Las prescripciones de la boda Canción seriada de boda

[pag.] 16 KANTIKA VIEJA DE BODAS /banios/ "dechémé paras para el banio ke me yeve ala agua yelada" * ( 2 estrofas completas +2 abreviadas, con estribillo) El dinero para el baño Canción lírica de boda

[pag.] 17 KANTIKA VIEYA DE BODAS/banios/ "la novia si foe al banio, si pero, si pero" (3 estrofas) $S F$ Canción lírica de boda 
[pag.] 18 KANTIKA VIEYA DE BODAS /banios/ "a primo kondé, primo kondé” * (4 estrofas completas + 5 abreviadas) Agua y jabón para el baño Canción lírica de boda

[pag.] 19 KANTIKA DE BODAS “a signor botikario kara de luna” (2 estrofas completas + 3 abreviadas) El boticario y la novia Canción seriada de boda

[pag.] 20 KANTIKA DE BODA “debacho de oyas vedres metieron las mezas" (4 estrofas completas) Debajo de línea separadora se agrega: "yo le mandi ala mi novia ouna fez dali"( 1 estrofa completa) $S F+$ Los presentes de los novios Canción lírica de boda + Canción seriada de bodas

[pag.] 21 KANTIKA DE PARIDAS “ke bien empleadas foeron las dolores” * (2 estrofas completas +3 abreviadas, con estribillo) El parto feliz Canción lírica de parida

[pag.] 22 KANTIKA VIEYA PARIDAS “debacho de ojas vedres metieron las mezas” (4 estrofas completas) El dolor del parto Canción lírica de parida

[pag.] 23 KANTIKA VIEYA DI HATANA /simnat tora/ "el bet amikdach ya lo estan fraguando" * (6 estrofas completas) La fragua de la Casa Santa Copla

[pag.] 24 KANTIKA VIEYA POR AVRAM AVINO "el era pastor de kavras con palavras" * (3 estrofas completas + 2 abreviadas, con estribillo) El engaño del amor Canción lírica de boda

[pag.] 25 KANTIKA VIEYA "viendo al boiey vindo al arado" (2 estrofas completas + 1 abreviada) El conjuro del buey Canción seriada

[pag.] 26 KANTIKA VIEYA/de sara de la tafsara/ "saro la mi saro toma mi konsejo" (2 estrofas completas, con estribillo) $S F$ Canción seriada

[pag.] 27 KANTIKA VIEYA "Metio el doktor la mano en la kavesa" * (2 estrofas completas +1 abreviada, con estribillo) El médico aprovechado Canción seriada

[pag.] 28 KANTIKA VIEYA DE /behor beni/ "por la kalejika del bakaliko mouevo" (5 estrofas completas) $S F$ Canción narrativa

[pag.] 29 ROMANSA "una notché al lunar me salí a passéar" * (6 estrofas completas) El seductor del casino + La princesa y el bozagi Canción narrativa + Romance

[pag.] 30 KANTIKA VIEYA /de bohor hadjopoglu/ "anicudja la zeli por vos foué esté rezilik" ( 3 estrofas completas, con estribillo) $S F$ Canción lírica

[pag.] 31 KANTIKA DE KOSTAN 1878 "ya mos vienen medikos mouevos" (4 estrofas completas, con estribillo) $S F$ Copla

[pag.] 32 KANTIKA OYIDA DE YAFA DE ADJI AVRAM 1876 "ouna notche yo me armi por voestro vizindado" (5 estrofas completas) El vecindado $+S F$ Canciones líricas

[pag.] 33 KANTIKA OYIDA DE JAFA DE HADJI AVRAM "koutro anios ke esto en prezion" (6 estrofas completas) El prisionero enamorado + El beso en la mezuzá Canciones líricas

[pag.] 34 KANTIKA OYIDA DE LOS PLEVNALIS 1878 "haim mordo avrio fabrika moeva" (7 estrofas completas) $S F$ Copla

[pag.] 35 ROMANSA DE SALONIQUE - OYIDA DE LOUNA KOHEN EN FILIBE 1882 "ouna mossa ke yo amo es ijika del amor" (10 estrofas completas) La escala de cabellos + La cama alta + Los peligros del mar + El gusano en la manzana + SF Canciones líricas 
[pag.] 36 ROMANSA OYIDA DE HAIM BEN NOUN BROUSSA "si tenech sintido moujer i marido" (6 estrofas completas) $S F$ Canción lírica

[pag.] 37 KANTIKA DE LOS FILIBILIS 1878 "tres ermanikas eran tres ermanikas son tra... “* (4 estrofas completas, con estriillo) Hero y Leandro (ó) Romance

[pag.] 38 KANTIKA DE LOS FILIBILIS OYIDA DE KLARA DEL HAZAN "notches boenas $/ 2 /$ notches son de namourar $/ 2 / " *$ (5 estrofas completas) Melisenda insomne $(+E l$ debate de las tres hermanas) Romance (+ Canción narrativa)

[pag.] 39 KANTIKA DE LOS PHILIBILIS OYIDA DE SARA I DOUDA DE LOUPO 1878 "arvolera arvolera de amade" * (8 estrofas completas) La vuelta del marido (í) Romance

[pag.] 40 ROMANSA. OYIDA DE YEHOUDA GUERCHON 1886 "ouna moutchatcha koando se empessa a engrandisser" (5 estrofas completas) El galanteo en el balcón Canción lírica

[pag.] 41 KANTIKA TOUNA YALISSI. DE SABETAY BIDJERANO broussa "ir me kero la mi madré, por estos moundos endelantré" * (7 estrofas completas) En busca del padre + La choza del desesperado Romances

[pag.] 42 ROMANSO OYIDO EN EDERNE 1886 "dies i otcho anios tengo la flor de la manseves" * ( 1 estrofa completa +6 abreviadas, con estribillo) La declaración del mancebo Canción narrativa

[pag.] 43 [+ 43 bis] ROMANSA OYIDA EN PHILIBE 1879 EN LA BODA DE MENAHEM LEVY "los ojos ke me miren ensiendem mi amor" ( 1 estrofa completa +9 abreviadas $S F$ Canción lírica

[pag.] 44 LA KANTIKA DE LOS ANIMALES "si veriach la poulga, amassando la massadoura" * ( 1 estrofa completa +4 estrofas abreviadas, con estribillo) La visión de los animales Canción seriada

[pag.] 45 KANTIKA DE PACHAROS /aves/ OHIDA DE VIZINO RAHAMIM BEHAR CHELOMO "ke kriyava la vieja eya kriyava un pouyiko, miteldé por nombré * (1 estrofa completa +5 abreviadas) La vieja y sus aves Canción seriada

[pag.] 46 KANTIKA DE LA GUERRA 1878 OYIDA DE OOUTCHA LA KRIADERA DE YOHANAM "russia russia ke es tou fantazia" (6 estrofas) $S F$ Copla

[pag.] 47 KANTIKA DEL FELEK 1878 OYDA DE OROUTCHA LA CRIADERA DE YOHANAN "oyid complas vente i ouna" (9 estrofas completas) SF Copla

[pag.] 48 KANTIKA DEL FELEK 1878 /oyida de lea yakob sid/ "elindé makara, si te arapas kapara, te arogas azer pas" ( 3 estrofas completas +4 abreviadas, con estribillo) $S F+L a$ bulisa zapateta Canción lírica

[pag.] 49 ROMANSA DE KOSTAN/ESKRITA DE RAHAMIM "tchikitica de la alhavaka, grandé de los ermanos" (2 estrofas completas +3 abreviadas) $S F$ Canción seriada

[pag.] 50 LA KANTIKA DE LA ESKOLA $1876 /$ kompozada de signor moche alkalay/ "mountchas grassias daremos, al dio bendiziremos" (4 estrofas completas) $S F$ Himno

[pag.] 51 KANTIKA NATIONALA /kopiado de eli sidi/ "asta koando malo igoismo" (4 estrofas completas) $S F$ Himno 
[pag.] 52 KANTIKA /eskrita de jakob arouété/ "las estreas en los sielos, i ouna i otra dos" (6 estrofas completas) La firmeza de las estrellas + El enamorado de noche + El enamorado del aire $+S F$ Canciones líricas

[pag.] 53 KANTIKA NATIONAL /eskrita de eli sid./ "onraremos, loaremos al kreador del moundo" ( 3 estrofas completas + 1 abreviada) $S F$ Himno

[pag.] 54 KANTIKA KOPIADA DE ELI SID "pasen loores, vengan kalores" ( 1 estrofa completa +1 abreviada) $S F$ Himno

[pag.] 55 [continúa en pág. 57] KANTIKA DE RIVKA DE CHILTON DE BROUSSA "altos sos i delikado, travadiko de kolor" (11 estrofas completas) SF Canción narrativa

[pag.] 56 KANTIKA OYIDA DE RIVKA CHILT DE BROUSSA "tres ermanikas eran, tres ermanikas son," * (8 estrofas completas) Hero y Leandro (ó) Romance

[pag.] 57 CONTINOUAR DE LA OJA 269 [véase pág. 55] "sounika estando en la pouerta" (3 estrofas completas) $S F$ Canción narrativa

[pag.] 58 ROMANSO ESPANOL DADO MEZO CHEMOUEL CHELOMO KALEV NASSIDO VIDIN MORODAR DE SOFIA AL INSRTITUT "vpjola la mi vojola ken bate tan demaniana" * (9 estrofas completas) La adúltera (á-a) (= La adúltera con un gato) Romance

[pags.] 59-60 KANTIKA ESKRITA DE RIVKA CHILTON EN BROUSSA 1888 “(...) la madrougada /avrich mé la mi linda dama" * (9 estrofas completas) La adúltera $(a ́-a)(=L a$ adúltera con un gato) Romance

[pags.] 61-62 KANTIKA ESCRITA EN BROUSSA "yo kiria ser rubissa assentada en la kuché" * (13 estrofas completas) $S F+$ Los peligros del mar Canciones líricas

[pag.] 63 ROMANSO ESCRITO DE RAHAMIM "tou paga mas del angel i la nouvé" (4 estrofas completas) $S F$ Canción lírica

[pag.] 64 ROMANSO DE DEZESPERASSION /ohida de m-messouloutchade seran en broussa/ "en la solidad de la noutchada" (10 estrofas completas) $S F$ Canción lírica

[pag.] 65 ROMANSO OHIDO EN PHILIBE DE S.PASSI "oh, ke linda manseva ke tou te estas aziendo" * (12 estrofas completas) Amor y muerte Canción lírica

[pag.] 66 KANTIKA DE LA MADRE DE F.AVRAM ARIÉ /ps-a lés/ "cayos kantan cayinas bailan, los pouyikos azen palmas" * ( 1 estrofa completa +3 abreviadas) El gallinero de la boda + La boda de la pulga y el piojo (Contrafactum) Canción lírica + Canción seriada

[pag.] 67 KANTIKA OHIDA DE RAHAMIM "por amar ouna donzéatchika de poka edad" (4 estrofas comletas) $S F$ Canción lírica

[pag.] 68 kantika vieja ohida en broussa/de rivka chilton/ "el rey ke mountcho madrougava" * (14 estrofas completas) Landarico Romance

[pag.] 69 KANTIKA ESKRITA MEZ ORAHAMIM ARIÉ / en broussa/ "ouna vez vos konti kontiz iko" (4 estrofas completas) $S F$ Copla

[pag.] 70 KANTIKA DE KOSTAN / ENBIZADA DE MI SIGNORA MADRE/ "ay mana estourourou me se rerezvala" (3 estrofas) El cántaro roto Canción lírica

[pags.] 71-72 KANTIKA DEL FELEK “ijo de poka édad sienté mi dotrino" * (9 estrofas completas) Consejos a un muchacho Copla 
[pag.] 73 KANTIKA VIEJA "avrich me la linda dama” (6 estrofas completas, con estribillo) Blancaniña Romance

[pag.] 74-75 KOMPLAS DEL YELEK “oyid mis ermanos i meté en tino" (10 estrofas completas) $S F$ Copla

[pag.] 76 KANTIKA DE LA VAVA /V.DONA/ "me mando mi madré por ayré boulayer" (2 estrofas completas +1 abreviada, con estribillo) $S F$ Canción seriada

[pag.] 77 KANTIKA DE BROUSSA KOPYDA DE RAHAMIM “ke ofissio tenech parizé , ke ofissio tehech en mano"* (8 estrofas completas) El rapto de Elena Romance

[pag.] 78 KANTIKA ESKRITA DE MENAHEM NISSIM "la reyna estava lavrando, dil berlini, ey aman" (*) (5 estrofas completas, con estribillo) Por qué no cantas, la bella Romance

[pag.] 79 ROMANSA KOPIADA DE RAHAMIM "tou ke fouetes i vinites" (5 estrofas completas +2 abreviadas, con estribillo) El Villano vil Romance

[pag.] 80 KANTIKA ESKRITA DE m-MELOUNATCHI DE SEMAN DE YAMBOL "vengach en bouena ora signora del vouestro kamino" * ( 2 estrofas completas + 4 abreviadas, con estribillo) La novia galana Canción lírica de boda

[pag.] 81 KANTIKA DE M-MELOUNATJI DE SEMAH "alta alta va la louna, quando nassi la kryatoura"* (4 estrofas completas +8 abreviadas) La cantiga de las horas Canción seriada

[pag.] 82 KANTIKA ESKRITA DE M-ME LOUNATCHI DE SEMAH “a la entrada del kourtijo bezo mano de los vizinos" * ( 2 estrofas completas +3 abreviadas, con estribillo) $L a$ entrada de la novia Canción seriada de boda

[pag.] 83-84 KANTIKA ESPAGNOLA DEL DJONK OJA 117 DE MANUSCRITO de joseph B. Mevorah zonana "al dyo alto con sou gracya" * (11 estrofas completas) Noche de Alhad Copla

[pag.] 85 KANTIKA VIEJA DI BODAS /esposorio/ "yo boli di cielo en cielo" ([3] estrofas completas) El vuelo tras la novia + El baño de la judería Canción seriada de boda + Canción lírica de boda

[pag.] 86 KANTIKA DI BODAS KANTADA DI ISRAEL LEVY delantre el rabino d-r hananel "keda en boena ora madre boena" * ([2] estrofas completas) La despedida de la novia Canción seriada de boda

[pag.] 87 [sin título] "alta alta es la luna, coando nasé la kriatura" * (1 estrofa completa +11 abreviadas) La cantiga de las horas Canción seriada

[pag.] 88 [en búlgaro: 'Canción compuesta por Luria, director de la escuela Hebrea de Pazarchik y delegado de la Aliance Israelite en 1905'] "percigido d'el cruel enemigo" ([4] estrofas completas) $S F$ Himno

[pag.] 89 [sin título] "dourmete mi alma, dourmete mi vista" * ([4] estrofas completas) La mujer engañada Romance

[pag.] 90 KANTIKA VIEJA BODAS /amores/ "la palomba blanca del ojo mavi" * ([5] estrofas completas) El pájaro del amor + Las flechas del amor + La llamada a la morena + La nave por partir + La escalera prodigiosa Canciones líricas 
[pag.| 91 O, MI DIO "dos dimandas dimando di ti" ([4] estrofas completas) $S F$ Himno [pag.] 92 SCHIR ORDENADE SOVRE IONA in melodia "ia chema evioneha" "fuyido como lo avi" (7 estrofas completas, con estribillo) $S F$ Himno

[pag.] 93 NOTCHI DI FRUTAS "boenas notches mis signoris djinitores" ([4] estrofas completas) $S F$ Himno

[pag.] 94 KANTIKA DE BODAS /espozorio/ "yoboli de cielo en cielo"* (3 estrofas completas) El vuelo tras la novia + El baño de la judería Canción seriada + Canción lírica de boda

[pag.] 95 ENPYIGADO ANDÉ EL JIDIO “oyid mis signorisesta grasia de koza” (9 estrofas completas) $S F$ Canción narrativa o Copla

[pags.] 96-97 KONSEJOS I MAKGIMAS “en loke sos de tchika édad, kon mountcha passensia" * (12 estrofas completas) SF Canción narrativa o Copla

[pag.] 98 KANTIKA DE BODA "ouna ija tenija el rey" * (5 estrofas completas) El mancebo de los botones + El papagayo de la novia Canciones líricas de boda

[pags.] 99-100 EL RATON, EL PACHARO I EL GATO [(] EL RATON [):] "loke ke keris mi kerido" (9 estrofas completas) El ratón, el pájaro y el gato Poema traducido

[pag.] 101 EL SAVIO ZIKIN / [palabra en búlgaro] "eckoutchad los mis signoris" (4 estrofas completas) $S F$ Poema traducido

[pag.] 101 bis KANTIKA VIEJA DE BODAS “oy kaz ada la venez iana, kzon un mansevo de esta kaza” * ( 1 estrofa completa +1 abreviada, con estribillo) $S F$ Canción lírica de boda

[pag.] 102 KANTIKA VIEJA DE BODA /ACHUGAR/ "achugar de novia galana, a boen mazal ya la manda" * (3 estrofas completas, con estribillo) El ajuar cle la novia galana Canción lírica de boda

Ipag.] 103 KANTIKA VIEJA DE BODA /achugar/ "oyi dizir ke bodas ay por aki vengo aver" * (4 estrofas completas, con estribillo) Los augurios de la boda + El novio desprendido Canciones seriadas de boda

[pag.] 104 [sin título] "Por las kalejas ke tu yras" ([6] estrofas completas) SF Canción lírica

[pag.] 105 CAMINANDO I NAVIGANDO NAVIGUE A LA FORTOUNA "louvias cayen de los sielos" (5 + 4 estrofas completas, así numeradas) En busca del padre Romance

[pag. ] 106 KANTIKA DI BODAS / amores/ "kara de flor de boenas kolores" ([3] estrofas completas) La cara de la novia Canción seriada de boda

[pag.] 107 KANTIKA DI BODAS / amores/ "Pachariko moevo la flor ti vas" ([3] estrofas completas) El pájaro nuevo Canción seriada de boda

[pag.] 108 KANTADA DE ISRAEL LEVY DELANTRE EL RABINO D-R A. HANANEL Sofia mai 1947 "kantar kero una parsa" ([17] estrofas completas) Las edades del hombre Copla [pag.] 109 [sin título] "en subiendon ala punta de la montana" ([1] estrofa completa) Melisenda saliendo del baño Romance

[pag.] 110 EL DON JUAN "ke oficio tenech mansevico" * ([1] estrofa completa) El rapto de Elena Romance 
[pag.] 111 LA DISGRASSIDA ERMANA “AVIA UNA LINDA MOUTCHATCHA”* ([3] estrofas completas) El hermano infame Canción narrativa

[pag.] 112 [sin título] "no mi mires amaria" * ([5] estrofas completas) Las enseñanzas del amor + El gusano en la manzana + SF Canciones líricas

[pag.] 113 KANTIKA VIEJA BODAS /esposorio/ "desidme la galanica si mi kirech koanto vos kero" ([2] estrofas completas) $S F+$ Los quidusín de la boda Canciones líricas de boda

[pag.] 114 KANTIKA VIEJA DE BODAS /esposorio/ "ayi dibacho del manzanero, ayi una novia sin saver" ([1] estrofa completa + [1] abreviada) SF Canción lírica de boda

[pag.] 115 KANTIKA VIEJAS BODAS "YO PASSI POR VOESTRA POERTA ORAS DI MITNHA"* ([3] estrofas completas) Los pasos infructuosos + El vestido de la novia + La novia alcanzada Canciones líricas de boda

\section{ALGUNOS EJEMPLOS DE CORRELACIONES CON LA TRADICIÓN ORAL SEFARDÍ}

Veanse a continuación tres ejemplos tomados de la colección de KANTIKAS VIEJAS (a) $y$, junto a cada uno de éstos, una de las versiones recogidas de la tradición oral de los judíos sefardíes de Oriente (b): las dos primeras son canciones de boda grabadas a informantes de Bulgaria, y la tercera, una canción acumulativa de la tradición jerosolimitana, aprendida allí y grabada a un informante oriundo de Salónica.

Ejemplo No. 1: La espera del novio

No. la (KANTIKAS VIEJAS, pag. 2)

\section{KANTIKA VIEJA DE BODAS / kidouchim/}

1/ signora novia abachech abacho non poedo non poedo ke me estan tokando tokados de noya ya me estan tokando para el manseviko ke me esta asperando

2/ o signora novia abachech abacho non poedo non poedo ke me estan peynando peynados de novia ya me estan peynando etc.

3/ o signora novia abachech abacho non poedo non poedo ke me estan alheniando alhenyas de novia ya me esto alhenyando etc.

4/ trokados etc. 
No. 1 b (Cantada por Mazaltó Lazar, nacida en Mustafá Pashá, Turquía, y criada en Sofía, Bulgaria. Recogida por S. Weich-Shahak en Yaffo, el 4 de julio de 1977, NSA Y2091/18)

- ¡Ah, señora novia, abaǰs̀ abajo!

- No puedo, no puedo, que me 'stó peinando, peinado de novia para el mancebico.

—iAh, señora novia, abajes̀ abajo!

-No puedo, no puedo, que me 'stó vistiendo, vestido de novia para el mancebico.

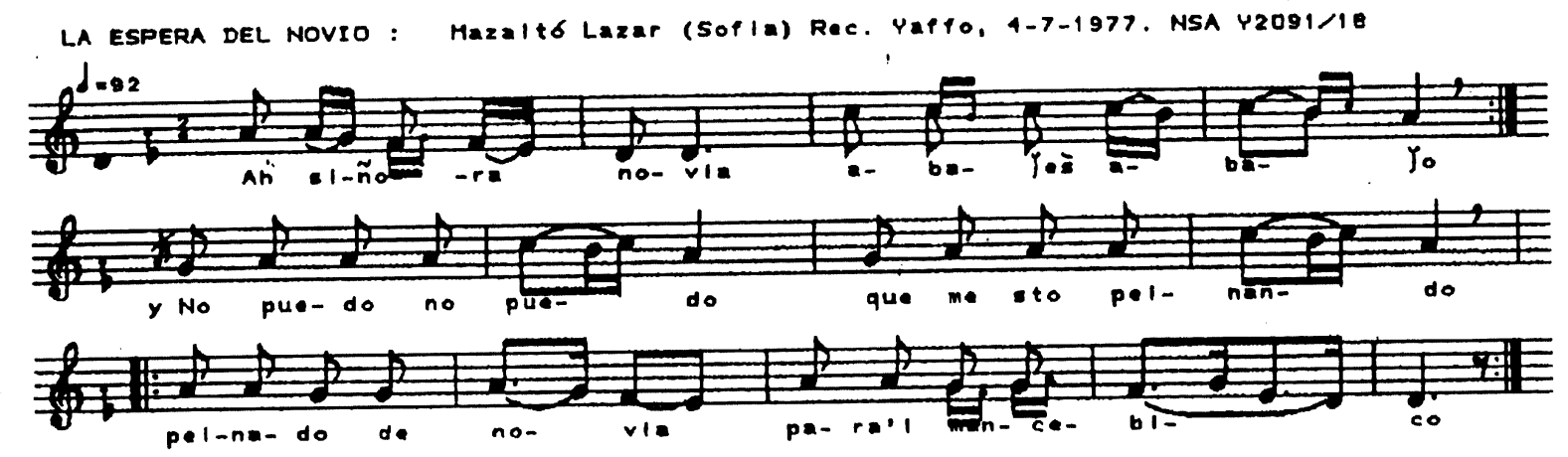

Ejemplo No. 2: La novia demudada

No. 2a (KANTIKAS VIEJAS, pag. 10)

VIEYA KANTIKA DE BODAS / houpa/

1. kouchiniko de alhavaka la kolor aresendera /2/

2. de onde vinich la mi novia, de ayi de onde emanuel

3. de ke estach tan demoudada, demoudese el mi amado

4. los kaveyos estan reboueltos, la kolor aresendida

5. i ariva i mas ariva ayi onde emanouel oun vaziko de klavo ahi estra en los bankos.

No. 2b (Cantada por Rachelica Levy, de Sofía, Bulgaria. Recogida por S. Weich-Shahak en Yaffo, el 23 de mayo de 1990, NSA Y5810a/10)

- ¿De qué estás tan demudada? (bis)

-Demudada del mi amado,

con un maćico de clavos.

- ¿Los cabellos tan reṿueltos? (bis) 
- Revueltos del mi amado, con un maćico de clavos.

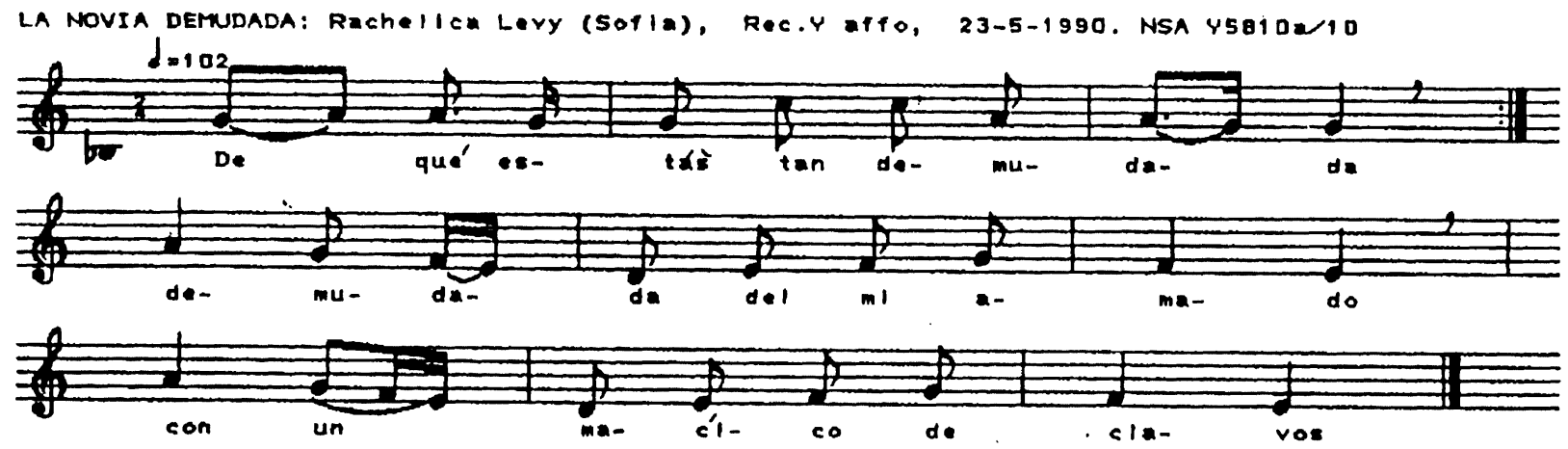

Ejemplo No. 3: La vieja y sus aves

No. 3a (KANTIKAS VIEJAS, pag. 45)

KANTIKA DE PACHAROS /aves/ OHIDA DE VIZINO RAHAMIM BEHAR CHELOMO

1. ke kriyava la vieja eya kriyava oun pouyiko, mitelde por nombre los pouyikos, yomtovikos, i la vieja soula vieja vieja sara, vieja ke mal tiené ke namorados kere.

2. ke la vieja eya kiriya ouna cayina, la gayina boula djamila, los pouyikos yomtovikos i la viela boula viela, viela sara,...etc.

3. ke keria la vieja, eya keriya i oun cayiko, metelde el nombre haham brago, la gayina boula djamila los poyikos...etc.

4. ke keria la vieja? eya keria ouna pata metelde el nombre i la pata boula sapata, el gayo haham brago, la gayina boula djamila etc.

5. ke kiria la vieja, eya kiria i oun pato metelde el nombrr, i el pato molo flako i la pata boula sapata...etc. 
6. ke 'kiria la vieja, eya kiria i oun bibo metelde por nombre i el bibo solo bibo i el pato molo flako...etc.

No. 3b (Cantada por Itshaq Simha, de 81 años de edad, nacido en Salónica, emigrado a Jerusalén, donde aprendió la canción. Recogida en Jerusalén el 28 de febrero de 1990).

La vieja, la vieja que mantiene, dos namorados tiene.

Tẻnía ella un pollico, ¿qué nombre que le metiera?

Le metía Sintuvico, y al pollico Sintuvico.

La vieja, la vieja que mantiene, dos namorados tiene.

Tenía ella una gallina, ¿qué nombre que le metiera?

Le metía Bula $\hat{\jmath}$ amila, y a la gallina Bula Jamila, y al pollico Sintuvico.

La vieja, la vieja que mantiene, dos namorados tiene.

Tenía y un gallo, ¿qué nombre que le metiera?

Le metía Haham Bravo, $\mathrm{Y}$ al gallo Haham Bravo, y a la gallina Bula famila, y al pollico Sintuvico.

La vieja, la vieja que mantiene, dos namorados tiene.

Tenía y un pato, ¿qué nombre que le metiera?

Le metía Sus Que Te Mato. Y al pato Sus Que Te Mato, y al gallo Haham Bravo, y a la gallina Bula Tamila, $y$ al pollico Sintuvico.

La vieja, la vieja que mantiene, dos namorados tiene.

\section{A MODO DE CONCLUSIÓN}

Es evidente que la deseable futura edición crítica de la colección de KANTIKAS VIEJAS será una labor compleja que deberá incluir concordancias tanto con fuentes publicadas como documentales, y de textos como de músicas. Asimismo deberá anotar y llamar la atención sobre toda la problemática de las lecturas dudosas, inexactas o claramente erróneas que se aprecian en el manuscrito, proponiendo las correspondientes restauraciones o soluciones. Igualmente deberá llegarse a una filiación más completa de todos los temas y a una caracterización más profunda de sus aspectos genéricos, ocasionales y funcionales. En tanto se alcanzan estas metas, para las que lo ideal sería la labor interdisciplinaria de un equipo formado por filólogos, etnomusicólogos y antropólogos, espero que este avance catalográfico y esta breve presentación de nuestra colección sirva de estímulo para conocer un documento de gran importancia sobre la poesía y la cultura de los sefardíes de Oriente. 
LA VIESA

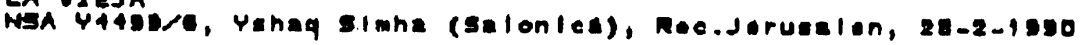
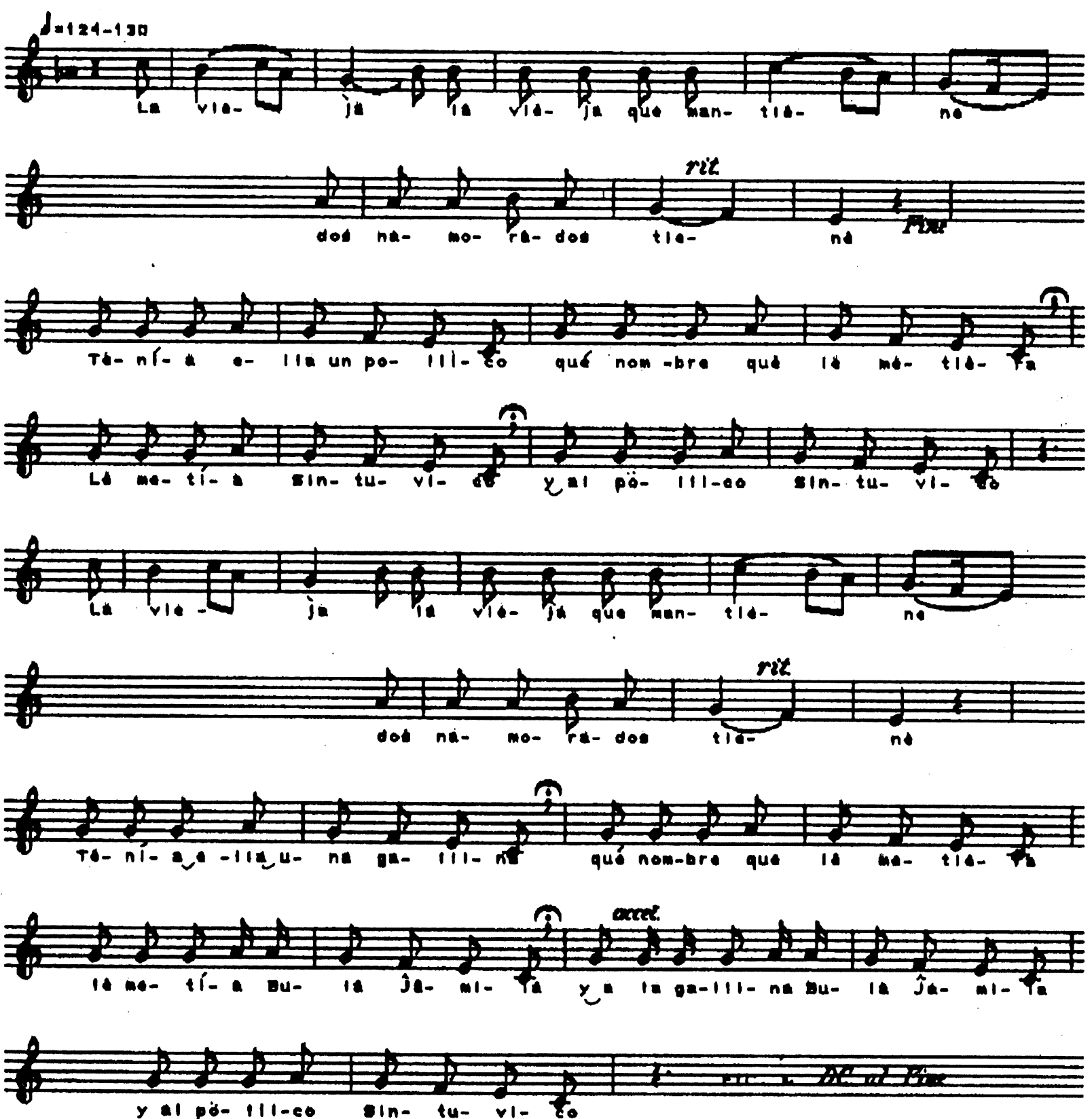